\title{
MOBILITY-AWARE ADAPTIVE COUNTER-BASED FORWARDING ELIMINATION TO REDUCE DATA OVERHEAD IN MULTICAST AD HOC ROUTING*
}

\author{
Carmen M. Yago Sánchez, Pedro M. Ruiz, and Antonio F. Gómez Skarmeta \\ Dept. of Information and Communication Engineering, University of Murcia, \\ Campus de Espinardo, 30100 Murcia, Spain \\ \{carmen,pedrom,skarmeta\}@dif.um.es
}

\begin{abstract}
Most of the previous efforts regarding multicast routing in MANETs, have been devoted to the provision of low-control overhead protocols, being able to maximize the packet delivery ratio. In multicast routing, the non-optimality of the forwarding structure can also lead to transmission of additional data packets (compared to the minimum required). We call those additional data packets data-overhead. In this paper, we present a counter-based forwarding elimination scheme, being able to reduce that overhead depending upon the mobility of the nodes. Our results show that this approach is able to enhance the bandwidth consumption of mesh-based multicast ad hoc routing protocols while maintaining nearly the same packet delivery ratio.
\end{abstract}

Keywords: ad hoc, routing efficiency, multicast, data overhead.

\section{Introduction and Motivation}

Nowadays mobile and wireless technologies are responding to the necessity of communicating everyday and everywhere without restrictions. In this atmosphere, ad-hoc networks, the infrastuctureless wireless networks, are gaining momentum. These networks are creating a big interest due to the variety of applications they have in different environments, for example rescue operations, battlefields, or communication between home automation devices.

There is also a manifest interest in allowing these networks to make use of multicast communication. Several routing protocols have been developed to route multicast traffic. These protocols are basically grouped into three

\footnotetext{
* This paper has been partially funded by the Spanish MCYT by means of the SAM (TIC2002-04531C04) project, and the "Ramon y Cajal" workprogramme and also by the "Consejeria de Trabajo, Consumo y Politica Social de la Region de Murcia" and the European Social Fund by means of the "Integrated Operacional Programme for the Region de Murcia 2000-2006".
} 
categories [Cordeiro et al., 2003]: stateless multicast like DDM, tree based protocols like MAODV or AMRIS and mesh based protocols like ODMRP or CAMP. There are also some hybrid approaches like AMRoute. Stateless multicast protocols are oriented to small groups. Tree based protocols do not have this restriction but they usually have problems with high mobility networks. In that case, they are outperformed by mesh based protocols which introduce more redundancy and alternate paths.

Mesh based approaches seem to be a good way to route multicast traffic in ad hoc networks, however they produce a considerable overhead. This overhead has two causes: firstly, the instability of the network makes it necessary to flood control messages periodically. Secondly, there is data overhead. Data overhead is a consequence of the non-optimality of multicast trees and meshes. Multicast meshes provide robustness but also cause forwarding nodes to redundantly transmit the same message in the same area. Then, as defined in [Ruiz and Gómez-Skarmeta, 2004] this data overhead consists of the data messages unnecessarily transmitted due to the redundancy of the mesh. In addition, provided that data traffic rates are higher than control packet rates, data overhead becomes the main source of sub-optimality of routing protocols, producing excessive bandwidth consumption, increased link-layer contention, and most of the issues associated to blind flooding [Ni et al., 1999].

Ruiz [Ruiz and Gómez-Skarmeta, 2004] showed that the problem of computing the minimal data-overhead multicast tree is an NP-complete problem. So, an approximation algorithm is necessary to limit the number of data messages delivered into the network.

Several algorithms and protocols have been developed to limit the number of messages flooded or multicasted into the network. These proposals can be basically grouped into two categories [Yi et al., 2003], topology based approaches and heuristic based approaches. The former refers to the use of topological information to reduce the number of nodes which can retransmit a message for example neighbour-topology-based schemes such as self-pruning [Lim and Kim, 2000] and multipoint relay MPR [Laouiti et al., 2001] or hierarchical approaches such as Domain-Based [Gui and Mohapatra, 2004] and OverlayDriven hierarchical multicast. The latter, rather than reducing the number of nodes which can retransmit, allows every node which is in charge of forwarding the message to decide, using a heuristic, whether to retransmit it or not; for example in [Ni et al., 1999] three schemes are defined to reduce the Broadcast Storm Problem in flooding: counter-based, distance based and probabilistic.

We consider a heuristic approximation algorithm based on the idea that limiting the number of redundant data messages without pruning multicast meshes will improve the efficiency whilst keeping the robustness. Within the heuristic category we encounter the counter-based algorithm particularly remarkable due to its performance, its ease of calculation and low resources consumption. 
This algorithm not only is useful to deal with overhead in flooding but also to make the nodes belonging to the mesh reduce the data overhead.

It is based on the "expected additional coverage" concept [Ni et al., 1999]. Every time a message is retransmitted by a node it covers an area in which the message can be heard. Then the "expected additional coverage" is the new area that would be covered for the first time if the node retransmitted the message. This area gets smaller every time a node listens to the message it has to retransmit because part of it is already covered by the message heard.

The counter-based algorithm proposes that a message must not be retransmitted if the host has heard it $C$ times before (from now on $C$ is called the threshold value), when the "expected additional coverage" becomes too small.

Using this counter-based approach, the threshold value $C$ has to be carefully chosen and it has to be a trade off between the performance of the protocol and the reduction of the overhead. A greater threshold provides better performance, but also higher overhead. Moreover, when the links are instable the number of messages heard has less significance because the instability of the links may have prevented the other nodes in the area from receiving the message. This might be solved by using a greater fixed threshold $C$, but then the message saving would decrease with the consequent increment of the overhead and its problems.

Due to these disadvantages, we consider the traditional counter-based scheme can be improved by making the threshold $C$ dependant on the local network conditions. To do this, we need a representative and distributed metric which tells the node about the stability of the network around it.

Based on this, we propose here a method to reduce the data overhead in mesh based protocols: the mobility-aware counter-based algorithm. It is a new adaptation of the counter-based algorithm which adapts itself to network conditions using a new mobility metric which fits the requirements detailed above. This metric, the modal link duration interval, is based on the stability of the links and allows the node to be aware of its local network conditions.

This paper is organized as follows, in the next section we propose the mobilityaware counter-based algorithm, our adaptation to multicast of the counterbased mechanism. In Section 3 we present and evaluate the results of the simulation. Finally, the conclusions are provided in the last section.

\section{Mobility-aware Counter Scheme}

As we have seen before, counter-based mechanisms [Ni et al., 1999], based on the "expected additional coverage" concept have been revealed as a good approach to reduce the redundancy in flooding. We use a similar approach for dealing with data overhead reduction. Below, we define our proposed metric, and afterwards we will describe our proposed approach. 


\section{Modal link duration interval (MLD) mobility metric}

We have chosen a metric based on the link duration [Boleng et al., 2002] which is a good indicator for protocol performance. The link duration metric fits many of our requirements: it is distributed and reflects network conditions. But if a few nodes behave very differently from the rest, the average may not reflect the behaviour of the majority of the network. We desire a metric which reflects the behaviour of the majority of the forwarder neighbours, hiding the distortion produced by "rebel" nodes. Thus, instead of the mean, we have adopted a modal interval of the average link duration of every forwarder neighbour.

The way we compute the metric is the following: firstly, we split the set of real numbers into intervals (for example, due to the heuristic function proposed the $x$ axis has been split into three intervals). Secondly, for a period of time $T$, every node computes the average link duration $L D_{f}$ with each forwarder neighbour it has. In order to compute if the links are up or down, the period of time $T$ is divided into $k$ timeslots whose size $t$ has to be long enough to allow the node to receive at least one message from every neighbour (this happens at least every periodic flooding timeout). Then, the average link duration for each forwarder node $f$ in a period of time $T, L D_{f}$, is defined as:

the $h(i)_{f}$ function determines if the link with $f$ is alive in the timeslot $i$.

$$
h(i)_{f}=\left\{\begin{array}{l}
1 \text { if the node has heard a message from } \mathrm{f} \\
0 \text { otherwise }
\end{array}\right.
$$

$C h_{f}$ calculates how many times the link goes up in the period $T$.

$$
C h_{f}=\left\{\begin{array}{l}
\sum_{i=1}^{i=k-1} \overline{h(i)} * h(i+1) \text { if } h(1)=0 \\
1+\sum_{i=1}^{i=k-1} \overline{h(i)} * h(i+1) \text { if } h(1) \neq 0
\end{array}\right.
$$

Then $L D_{f}$ is calculated by dividing the time in which the link with node $f$ is alive over the times the link goes up in the period $T$ :

$$
L D_{f}=\left\{\begin{array}{l}
\sum_{k} h(i)_{f} \text { if } C h_{f}=0 \\
\frac{\sum_{k} h(i)_{f}}{C h_{f}} \text { if } C h_{f} \neq 0
\end{array}\right.
$$

Thirdly, every computed $L D_{f}$ belongs to an interval from the set previously defined in the first step, then, modal link duration interval $M L D$ is the interval to which the greatest number of $L D_{f}$ belongs.

In order to adapt the metric asymptotically to the changing network conditions, the $T$ period has a window structure computing the metric every $t$ time units. 
This metric gives the node an idea of what is happening with the majority of the nodes in the neighbourhood, what is happening in the area it is in. For example, if $M L D$ is higher, the network around is basically stable, no matter if there are a few nodes with low stable links or they are moving together in the same direction.

\section{The Mobility-aware Counter-based Algorithm}

The mobility-aware counter-based algorithm used to reduce data overhead in mesh based protocols is built around the basic counter algorithm. This mobility-aware counter-based algorithm is only executed by the nodes belonging to the mesh because they are in charge of forwarding the data messages and consists of two processes working concurrently: the first computes the value of the threshold $C$ as a function of $M L D, C=C C(M L D)$, whereas the second applies the counter-based approach. Fig. 1 shows how the mobilityaware counter-based algorithm works and we have chosen a function $C=$ $C C(M L D)$ based on the following heuristics:

- If the value of $M L D$ is low, the node itself has not been able to establish stable links with other nodes. Probably, when it is going to resend the message, the links with the intended receivers will be broken. For this reason, it will only retransmit the message if it has heard it very few times, to deal with the possibility that there are hardly any nodes in the area able to forward the message.

- If the value of $M L D$ is medium, the node is in a network where a great number of the nodes are able to set links of a moderate duration. A medium value of $C$ helps to cover the expected area.

- If the value of $M L D$ is high, the network is basically stable: there are few changes, so a low threshold should be enough.

\section{Simulation Results}

The data overhead reduction mechanisms presented above are going to be applied to a multicast routing protocol to test if they really assess the performance of the proposed approach. We have chosen ODMRP because it is a well known mesh based protocol and offers a good performance when compared with other multicast routing protocols [Lee et al., 1999].

To simulate our mobility-aware counter scheme we have considered the following time ranges:

- $M L D$ is the interval $[0,21)$ : it has a low value. Then $C$ is low, and we considered low $C=2$. According to [Boleng et al., 2002] links 


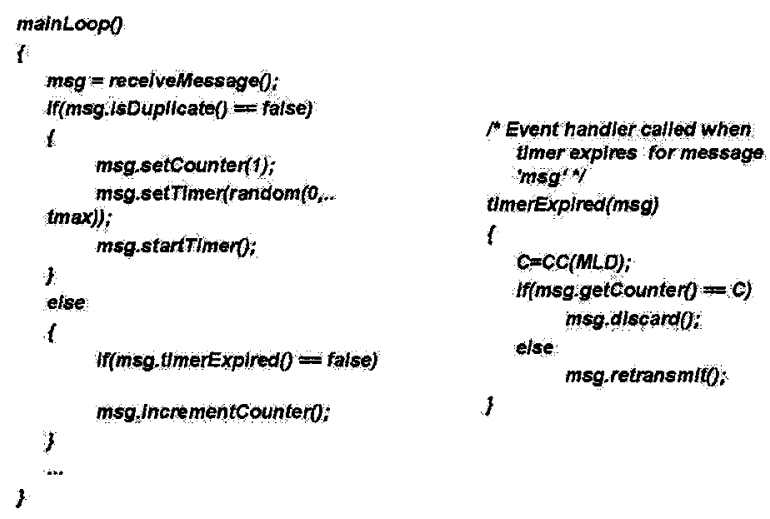

Figure 1. Pseudocode for the mobility-aware counter-based algorithm

with a lifetime lower than 20 seconds lead to a poor performance of the protocols.

- $M L D$ is the interval $[21,75):$ it has a medium value. Then $C$ is medium and we considered medium $C=3$.Links which last more than $75 \mathrm{sec}-$ onds are highly stable links when dealing with the instability of ad-hoc networks.

- $M L D$ is the interval $[75, \infty)$ : it has a high value. Then $C$ is low, and we considered low $C=2$.

The period of time $T$ in which the $M L D$ is calculated is 90 seconds which we consider a good value to capture both long and short lived links. The $M L D$ is computed every 3 seconds because that is the flooding timeout. We have chosen $C=3$ as the medium value because according to [Ni et al., 1999], the additional expected coverage is about $10 \%$.

For simulating we have used the NS-2 [Ns] network simulator version $2.1 \mathrm{~b} 8$ with the multicast extensions developed by the Rice University Monarch Project [Mon].

The simulated scenario consists of 100 mobile hosts randomly distributed over an area of $1600 \times 1200 \mathrm{~m}$. The radio channel capacity for each mobile node is $2 \mathrm{Mb} / \mathrm{s}$, using the IEEE 802.11b DCF MAC layer and a communication range of $250 \mathrm{~m}$. Each one of the approaches has been evaluated over the same pre-generated set of 330 scenarios with varying mobility speed and traffic loads. Mobile nodes move using a Gauss-Markov model [Camp et al., 2002 ] with a maximum speed of $0,5,10,15$, and $20 \mathrm{~m} / \mathrm{s}$. Ten different traffic loads where tested consisting of 1,2 , and 4 CBR sources for the same multicast group, and a varying number of receivers, 5, 15, and 30 . 


\section{Performance Metrics}

To assess the effectiveness of the different mechanisms, we have used the following performance metrics:

- Packet delivery ratio. Defined as the number of data packet successfully delivered over the number of data packets generated by the sources.

- Normalized packet overhead. Defined as the total number of control and data packets sent and forwarded normalized by the total number of packets successfully delivered.

- Forwarding efficiency. The average number of times that a multicast data packet was forwarded by the routing protocol. This metric represents the efficiency of the underlying forwarding structure.

- Average delivery delay. For each receiver, the average delay of all packets received is computed. Then the average delivery delay is the mean of all of these averages.

\section{Analysis of the Results}

We have simulated three approximations of the counter-based scheme: two with fixed threshold values of $\mathrm{C}=2$ and $\mathrm{C}=3$ respectively and one with our mobility-aware counter approach. Their results have been compared with the results offered by the ODMRP protocol. Fig. 2 to fig. 7 show the results with 15 receivers and 1 , and 4 sources as a function of the maximum speed of the nodes. The increment of the number of sources implies the increase of both the traffic and the density of the forwarder nodes.

Fig. 2 and fig. 6 show the packet delivery ratio (PDR). In all simulations, the fixed threshold $C=2$ offers an insufficient PDR: almost always under $95 \%$. This is because having fewer retransmissions affects the network connectivity. Regarding the other approaches, counter-based schemes have worse PDR than ODMRP in sparse networks. This is because the mesh has not enough redundancy; this situation gets worse as speed increases because there are more link breakages and there are no alternative paths. As the network becomes denser, counter-based mechanisms offer a higher PDR and with $C=3$, the speed only causes a slight drop. However, our mobility-aware counter scheme gets a higher PDR when the speed is higher than $10 \mathrm{~m} / \mathrm{s}$. This is because our scheme adapts itself to higher speeds.

Fig. 3 and fig. 7 show the normalized overhead. In all cases, the use of counter-based schemes reduces considerably the overhead, obtaining the higher saving when more redundancy is in the network. Our mobility-aware counter scheme provides better savings than $C=3$ but worse than $C=2$ (when compared with ODMRP the saving is between $27 \%$ and $56 \%$ ). It also 
provides better overhead reduction when the mesh is denser. However, unlike fixed threshold approaches, there is a variation with the speed of the nodes: the saving is better in low mobility environments where the links are stable. This is because our scheme detects the stability of the network, and hence it can provide better savings.

Fig. 4 and fig. 8 show the forwarding efficiency (FEF). It is observed that the number of times a packet has to be forwarded experiments an important decrease compared with ODMRP when using counter-based schemes. This is because they prevent the nodes from retransmitting unnecessary broadcasts. Results are similar to those for overhead, and our mobility-aware counter scheme provides lower FEF than $C=3$ but greater than $C=2$.

Fig. 5 and fig. 9 show the average delivery delay. Here, all counter-based schemes present similar results higher than the ones presented by ODMRP. This is because ODMRP always follows a shortest path tree approach, but when using a counter-based approach the path that a message follows from its source to its destination does not have to be the shortest one, since there are nodes which do not retransmit.

Summing up, these graphs show that counter schemes can be applied successfully to multicast mesh based protocols. In general, all the adaptations save overhead and obtain a better forwarding efficiency, getting better results in environments where the density of forwarder nodes is higher. Regarding our mobility-aware counter scheme, its performance can be situated into both thresholds: it usually offers an acceptable PDR (near the PDR of the $C=3$ approach) with a better forwarding efficiency and overhead reduction. The overhead curve is nearer $C=2$ (whose performance has resulted insufficient) when the mobility is low because links last more time and it is nearer $C=3$ when the mobility is high because of link breakages. The best performance is when the mobility is low but not completely static, in which it offers really better PDR than $C=2$ but the overhead saving is almost the same.

\section{Conclusions}

The alternative paths grant robustness to multicast mesh based protocols at the expense of adding redundant data transmissions. Providing more efficiency to data dissemination has to be done while keeping the robustness. To address this question we have made the mesh nodes use the counter-based algorithm to decide whether to forward or not. This approach has proven to be a good solution to reduce data overhead while maintaining the performance. However, this approach is based on a fixed threshold value: if this value is low, the algorithm provides better savings at the expense of performing worse, and vice versa. For this reason we have proposed in this paper an adaptive variant of this approach. The proposed mobility-aware counter-based mechanism obtains 


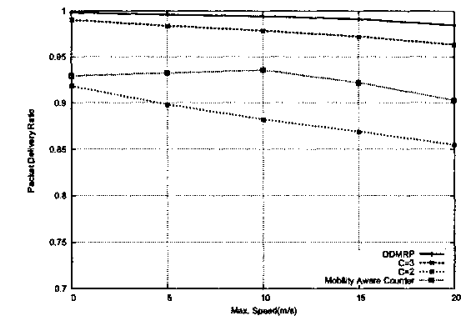

Figure 2. PDR with 1 source and 15 receivers.

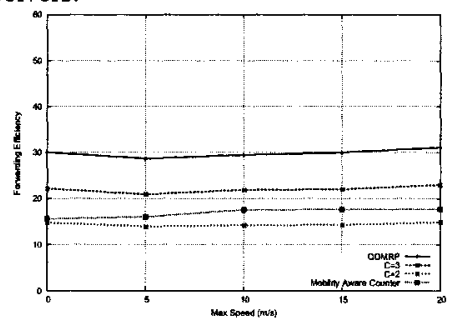

Figure 4. FEF with 1 source and 15 receivers.

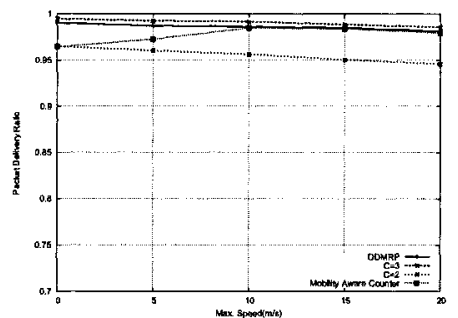

Figure 6. PDR with 4 sources and 15 receivers.

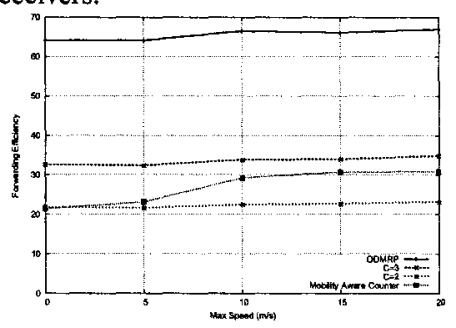

Figure 8. FEF with 4 sources and 15 receivers.

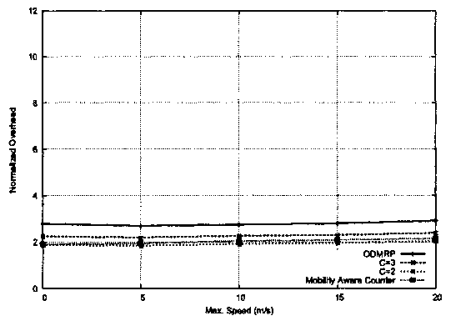

Figure 3. Overhead with 1 source and 15 receivers.

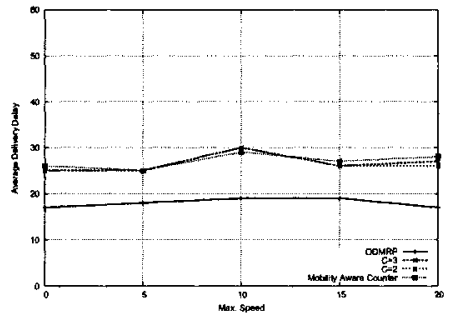

Figure 5. Average Delivery Delay with 1 source and 15 receivers.

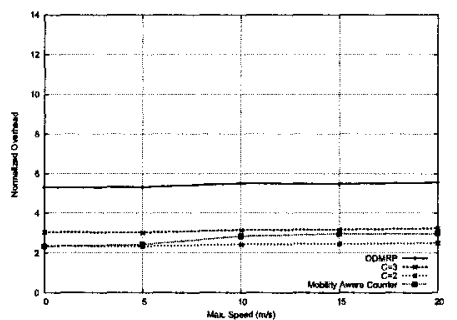

Figure 7. Overhead with 4 sources and 15 receivers.

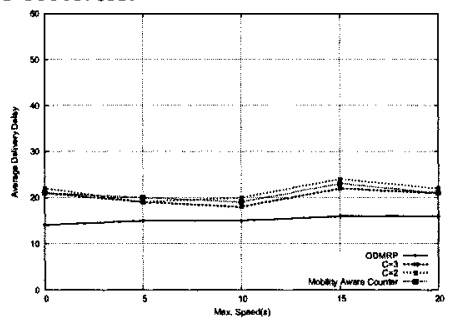

Figure 9. Average Delivery Delay with 4 sources and 15 receivers. 
good performance as high thresholds do, but has better efficiency in terms of bandwidth consumption. Using the mobility-aware counter-based mechanism, every forwarder node changes its threshold value according to the stability of the network around it. To allow the node to realize the network conditions, the algorithm uses a new metric, the modal interval of the link duration, which gives the modal interval of the average duration of the links the node has. This gives the node an idea of how the majority of the nodes around it are behaving. Using our mobility-aware counter-based mechanism, the mesh is not pruned, and as a consequence, its robust structure is preserved. The good performance of the protocol is kept and the overhead is reduced up to a $56 \%$.

\section{References}

[Ns] The Network Simulator Ns-2.

[Mon] The Rice University Monarch Project.

[Boleng et al., 2002] Boleng, J., Navidi, W., and T.Camp (2002). Metrics to enable adaptive protocols for mobile ad hoc networks. In Proc. of the International Conference on Wireless Networks (ICWN '02), pages 293-298.

[Camp et al., 2002] Camp, T., Boleng, J., and Davies, V. (2002). A survey of mobility models for ad-hoc network research. Wireless Communications and Mobile Computing (WCMC): Special issue on Mobile Ad Hoc Networking: Research, Trends and Applications, 2(5):483-502.

[Cordeiro et al., 2003] Cordeiro, C. Moreis, Gossain, H., and Agrawal, D. P. (2003). Multicast over wireless mobile ad hoc networks: present and future directions.

[Gui and Mohapatra, 2004] Gui, C. and Mohapatra, P. (2004). Scalable multicasting in ad hoc networks. In IEEE INFOCOM.

[Laouiti et al., 2001] Laouiti, A., Qayyum, A., and Viennot, L. (2001). Multipoint relaying: An efficient technique for fboding in mobile wireless networks. In 35th Annual Hawaii International Conference on System Sciences (HICSS'2001). IEEE Computer Society.

[Lee et al., 1999] Lee, S. J., Gerla, M., and Chiang, C.C. (1999). On demand multicast routing protocol. In Proc. of the IEEE WCNC'99, pages 1298-1302.

[Lim and Kim, 2000] Lim, H. and Kim, C. (2000). Multicast tree construction and fboding in wireless ad hoc networks. In Proc. of the $3 r d$ ACM international workshop on Modeling, analysis and simulation of wireless and mobile systems, pages 61-68.

[Ni et al., 1999] Ni, S.Y., Tseng, Y.C., Y.S., Chen, and Sheu, J.-P. (1999). The broadcast storm problem in a mobile ad hoc network. In Proc. of Int'l Conf. Mobile Computing and Networking (MOBICOM), pages 151-162.

[Ruiz and Gómez-Skarmeta, 2004] Ruiz, P. M. and Gómez-Skarmeta, A. F. (2004). Mobilityaware mesh construction algorithm for low data overhead in multicast ad hoc routing. Journal of Communications and Networks (JNC), 6(5):331-342.

[Yi et al., 2003] Yi, Y., Gerla, M., and Kwon, T. J. (2003). Efficient fboding in ad hoc networks: a comparative performance study. In Proc. of the IEEE International Conference on Communications (ICC 2003). 